\title{
Mechanisms and Clinical Significance of Pharmacokinetic-Based Drug-Drug Interactions with Drugs Approved by the U.S. Food and Drug Administration in $2017^{\text {⿷ }}$
}

\author{
Jingjing Yu, Ichiko D. Petrie, René H. Levy, and Isabelle Ragueneau-Majlessi \\ Department of Pharmaceutics, School of Pharmacy, University of Washington, Seattle, Washington \\ Received October 14, 2018; accepted November 12, 2018
}

\begin{abstract}
Pharmacokinetic-based drug-drug interaction (DDI) data for drugs approved by the U.S. Food and Drug Administration in $2017(N=34)$ were analyzed using the University of Washington Drug Interaction Database. The mechanisms and clinical relevance of these interactions were characterized based on information from new drug application reviews. CYP3A inhibition and induction explained most of the observed drug interactions (new drugs as victims or as perpetrators), and transporters mediated about half of all DDIs, alone or with enzymes. Organic anion transporting polypeptide (OATP)1B1/1B3 played a significant role, mediating more than half of the drug interactions with area under the time-plasma curve (AUC) changes $\geq 5$-fold. As victims, five new drugs were identified as sensitive substrates: abemeciclib, midostaurin, and neratinib for CYP3A and glecaprevir and voxilaprevir for OATP1B1/1B3. As
\end{abstract}

perpetrators, three drugs were considered strong inhibitors: ribociclib for CYP3A, glecaprevir/pibrentasvir for OATP1B1/1B3, and sofosbuvir/velpatasvir/voxilaprevir for OATP1B1/1B3 and breast cancer resistance protein. No strong inducer of enzymes or transporters was identified. DDIs with AUC changes $\geq 5$-fold and almost all DDIs with AUC changes 2- to 5-fold had dose recommendations in their respective drug labels. A small fraction of DDIs with exposure changes <2-fold had a labeling impact, mostly related to drugs with narrow therapeutic indices. As with drugs approved in recent years, all drugs found to be sensitive substrates or strong inhibitors of enzymes or transporters were among oncology or antiviral treatments, suggesting a serious risk of DDIs in these patient populations for whom effective therapy is already complex because of polytherapy.

\section{Introduction}

In the last few decades, the U.S. Food and Drug Administration (FDA) and the pharmaceutical industry have contributed to the development of a systematic, risk-based approach for evaluating pharmacokinetic (PK)-based drug-drug interactions (DDIs) and communicating the results to the scientific and medical communities. In the last 2 years, the approach recommended by the FDA has been updated (https://www. fda.gov/Drugs/DevelopmentApprovalProcess/DevelopmentResources/DrugInteractionsLabeling/ucm093664.htm FDA 2017a,b, 2018). These approaches are best expressed in new drug application (NDA) approval packages because they include preclinical and clinical investigational data of the new drugs and the implications of those findings in product labels. These NDA reviews are also useful because only a small portion of their data becomes available in the scientific literature, even later. Thus, NDA reviews provide a unique perspective on the evolution of drug-interaction science, acting like a snapshot of the implementation of DDI guidances and newer regulatory

https://doi.org/10.1124/dmd.118.084905.

S This article has supplemental material available at dmd.aspetjournals.org. recommendations in the mechanistic and clinical contexts of various therapeutic classes. An example is how the complex metabolismtransporter interplay affects our evolving understanding of the mechanism and potential clinical risk associated with PK-based drug interactions of new drugs. In that context, this article provides an analysis of the significant clinical DDIs associated with the 2017 NDAs and shows how to predict and manage possible DDI risk and administer these new drugs safely in certain patient populations.

\section{Materials and Methods}

This analysis was performed using the University of Washington Metabolism and Transport Drug Interaction Database and the Pharmacogenetics (PGx) Database (e-PKGene) (http://www.druginteractioninfo.org) according to the method previously described (Yu et al., 2014, 2016, 2017, 2018). Clinical DDI study results were obtained from dedicated DDI clinical trials, PGx studies, as well as physiologically based pharmacokinetics (PBPK) modeling studies that functioned as alternatives to dedicated clinical studies. Mean area under the timeplasma curve (AUC) ratios were the metric used to evaluate clinical studies, using $\mathrm{AUC}_{\text {inf }}$ unless otherwise noted. In the present analysis, all positive clinical studies, we analyzed AUC ratios $\geq 1.25$ for inhibition DDIs or PGx studies and $\leq 0.8$ for induction DDIs, including mechanistic and comedication evaluations. To allow a

ABBREVIATIONS: AUC, area under the time-plasma concentration curve; BCRP, breast cancer resistance protein; DDI, drug-drug interaction; FDA, Food and Drug Administration; NDA, new drug application; NME, new molecular entity; NTI, narrow therapeutic index; OATP, organic anion transporting polypeptide; P450, cytochrome P450; PBPK, physiologically based pharmacokinetics, P-gp, P-glycoprotein; PGx, pharmacogenetic (s); PK, pharmacokinetic(s); PXR, pregnane X receptor; UGT, UDP-glucuronosyltransferase. 
general comparison for all DDIs included in this analysis, any drug interactions with AUC changes $\geq 5$-fold, 2 - to 5-fold, or 1.25- to 2 -fold were considered strong, moderate, or weak inhibition or induction interactions, respectively, whether they are mediated by enzymes and/or transporters.

\section{Results}

A total of 34 NDAs [including one combination drug with two new molecular entities (NMEs), so total NME number $=35$ ] were approved by the FDA in 2017, and their chemical structures are presented as supplemental material (Supplemental Table 1). The most represented therapeutic areas were oncology drugs (26\%) and anti-infective agents (23\%) including four antibacterials, three antivirals, and one antiparasitic), followed by central nervous system agents (12\%) and metabolism disorder/endocrinology drugs $(12 \%)$. This representation pattern of therapeutic classes is similar to that observed with drugs approved from 2013 to 2016 (Yu et al., 2018). Among the nine new chemical entities approved for cancer treatment, seven were kinase inhibitors, suggesting that this therapeutic class has a predominant role as a cancer treatment target.

All the NDAs had in vitro and/or clinical drug metabolism and transport interaction data. Among them, 26 NDAs had clinical drug interaction data available, five presented PGx information, and six had PBPK simulation data. Approximately 150 clinical DDIs had positive results, including 61 inhibition DDIs (plus two PGx studies) and 29 induction interaction studies where NMEs served as substrates and 54 inhibition interaction studies and three induction DDIs where NMEs served as perpetrators. Given the large amount of information included in the NDA reviews, only the most significant DDIs with exposure changes $\geq 5$-fold are highlighted in detail in the following sections and presented in Table 1; the remainder of drug interaction data are briefly reviewed.

NMEs as Substrates of Enzymes. All 35 NMEs were assessed in vitro as substrates of specific drug-metabolizing enzymes. The numbers of NME substrates of drug-metabolizing enzymes are presented in Fig. 1A. As expected, CYP3A played a major role, metabolizing twothirds of the NMEs, followed by CYP2D6, CYP1A2, and the CYP2C families.

Not surprisingly, all inhibition DDIs with AUC ratios $\geq 5(N=$ 3 NMEs) were mediated by CYP3A under coadministration with ketoconazole, the standard strong CYP3A inhibitor (Table 1). Because of the large increases in drug exposure, labeling recommendations (avoidance, dose reduction, considering alternative therapies, and monitoring adverse reactions) were included regarding concomitant use with strong CYP3A inhibitors. Based on the FDA classification, abemaciclib, midostaurin, and neratinib were considered sensitive substrates of CYP3A. These three drugs are kinase inhibitors indicated for the treatment of breast cancer (abemaciclib and neratinib) or leukemia (midostaurin), suggesting a need to manage drug use carefully when these patients are also treated with CYP3A inhibitors.

Abemaciclib exhibited the largest exposure change, with up to a 16-fold increase predicted using PBPK modeling and simulations in healthy subjects upon coadministration with ketoconazole at a dose expected to produce $100 \%$ inhibition of CYP3A (FDA, 2017p). In vitro studies suggest that abemaciclib is primarily metabolized by CYP3A to several active metabolites. Because these metabolites are equipotent to the parent drug, the total analyte exposure (including abemaciclib and these active metabolites) and the relative potency-adjusted unbound exposure of abemaciclib plus these active metabolites were also used as markers of drug exposure by the sponsor. With ketoconazole, the total analyte AUC was predicted to increase 6.87-fold, and the potencyadjusted unbound AUC was increased by 2.87 -fold. A smaller change in the exposure was predicted or observed with coadministration of other strong CYP3A inhibitors. For example, itraconazole (dose to assume 90\% CYP3A inhibition) was predicted to increase abemaciclib AUC by 7.15-fold; the total analyte, AUC by 2.76-fold; and the relative potencyadjusted unbound AUC of abemaciclib plus its active metabolites by 2.20 -fold in healthy subjects using PBPK models. In a clinical study in which patients were administered clarithromycin $500 \mathrm{mg}$ twice daily for 14 days, abemaciclib AUC increased by 3.37-fold, 2.19-fold in total analyte AUC, and 1.70-fold increase in the relative potency-adjusted unbound AUC of abemaciclib plus its active metabolites. The differential effects of ketoconazole on the PK of abemaciclib in comparison with other strong CYP3A inhibitors may be due to several factors, including the nonlinearity of the AUC versus $[\mathrm{I}] / \mathrm{K}_{\mathrm{i}}$ relationship between $90 \%$ and $100 \%$ inhibition (Ito et al., 2004) and/or the differential effects of strong inhibitors on first-pass versus systemic CYP3A metabolism of abemaciclib (Boxenbaum, 1999). Similar behavior has been observed with midazolam, the sensitive substrate of CYP3A, where coadministration with ketoconazole (400 $\mathrm{mg}$ once daily for 5 days) increased midazolam AUC approximately 17-fold (Boulenc et al., 2016), whereas itraconazole (200 mg once daily for 4 days) and clarithromycin (500 mg twice daily for 7 days) resulted in relatively smaller increases (10.80- and 8.39fold, respectively) (Olkkola et al., 1994; Gurley et al., 2006). Given the large magnitude of exposure change in abemaciclib when coadministered with ketoconazole and the potential concerns for unknown offtarget toxicities related to increased abemaciclib exposure, concomitant use of ketoconazole with abemaciclib should be avoided. For other strong CYP3A inhibitors, a reduction in abemaciblib dose is recommended upon concomitant administration (FDA, 2017p). With the moderate inhibitors diltiazem and verapamil, the increases in exposure of abemaciclib and in the relative potency-adjusted unbound AUC of abemaciclib plus its active metabolites were both predicted to be low and not considered clinically meaningful.

Midostaurin undergoes extensive metabolism, primarily by CYP3A, to two active metabolites, CPG52541 and CPG62221. Concomitant administration of ketoconazole (400 $\mathrm{mg}$ once daily for 10 days) with a 50-mg single dose of midostaurin in healthy subjects increased the AUC of midostaurin and CGP62221 by 10.42- and 3.51-fold, respectively, and increased the $\mathrm{AUC}_{0-120 \mathrm{~h}}$ of CGP52421 by 1.21 -fold $\left(\mathrm{AUC}_{\mathrm{inf}}\right.$ of CGP52421 was not evaluated owing to the its long elimination half-life of 482 hours) (Dutreix et al., 2013; FDA 2017m). When multiple doses of midostaurin (50 or $100 \mathrm{mg}$ twice daily for 28 days) were coadministered with itraconazole (100 mg twice daily for 13 doses) in patients, on day 28, the $\mathrm{AUC}_{\text {tau }}$ of midostaurin and CGP52421 was increased by only 1.63 - and 1.20 -fold, respectively, and the $\mathrm{AUC}_{\text {tau }}$ of CGP62221 decreased by $13 \%$. The $C_{\min }$ values on day 28 for midostaurin, CGP62221, and CGP52421 were increased by 2.1-, 1.2-, and 1.3-fold, respectively. Of note, midostaurin exhibited timedependent PK, with an initial increase in $C_{\min }$ reaching its highest value during the first week, followed by a decline to a steady state after approximately 28 days of administration. The PK of CGP62221 showed a similar behavior; the plasma concentrations of CGP52421 continued to increase after 1 month of treatment. According to the sponsor, this result is possibly due to autoinduction of CYP3A as in vitro studies showed that both midostaurin and the two metabolites had the potential to induce CYP3A at clinically relevant concentrations (FDA, 2017m). The PK profile of midostaurin explains why the effect of strong CYP3A inhibitors on the PK of midostaurin appears to be dependent on the duration of midostaurin dosing, with a 10-fold increase in exposure observed after a single-dose administration of midostaurin and a 2-fold increase observed at steady state. Since midostaurin is used at multiple doses in cancer patients and considering the 2 -fold increase observed in midostaurin exposure at steady state, monitoring for increased risk of 


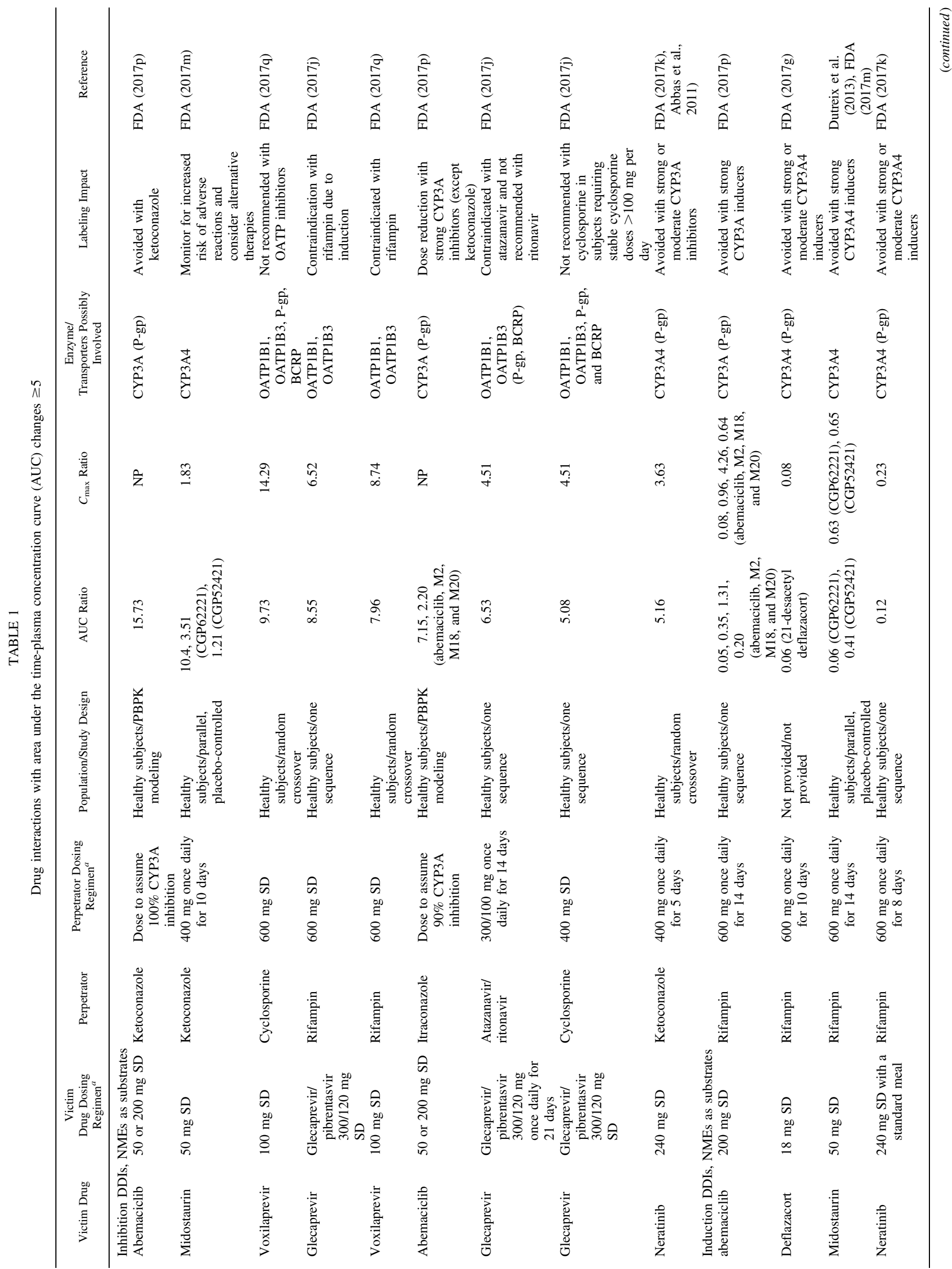

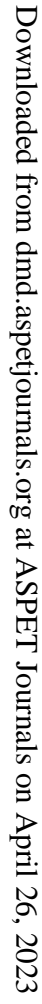


Yu et al.

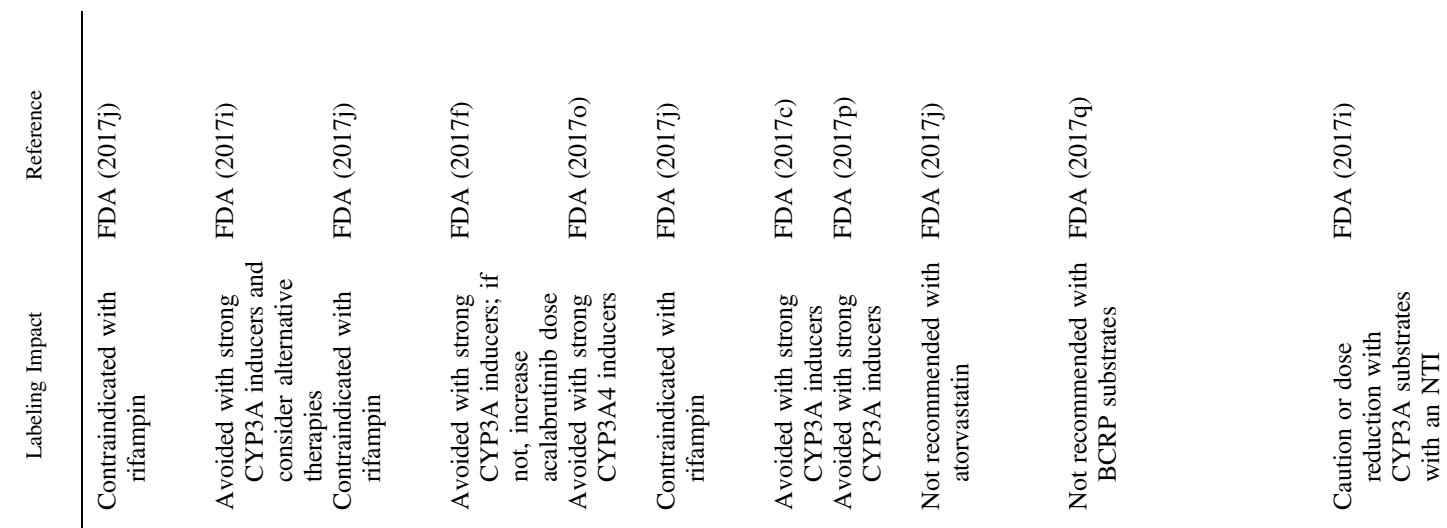

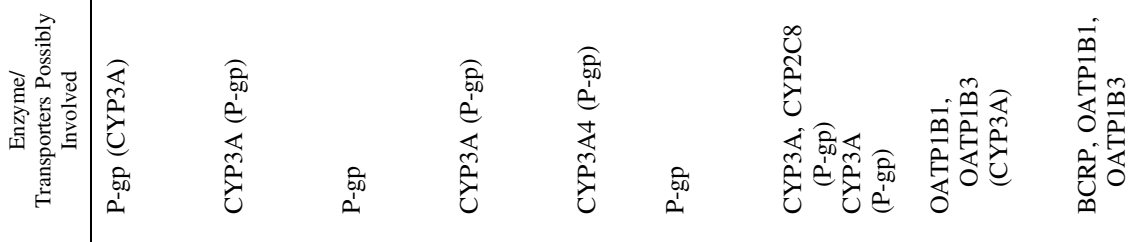

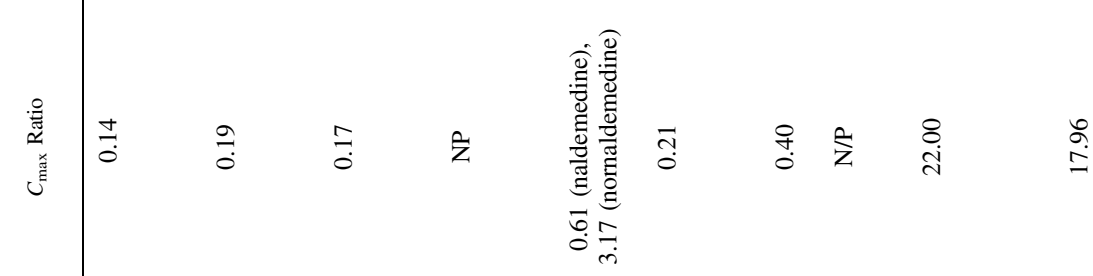

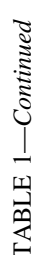

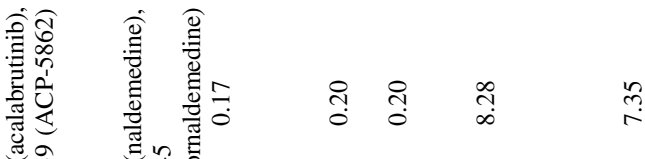

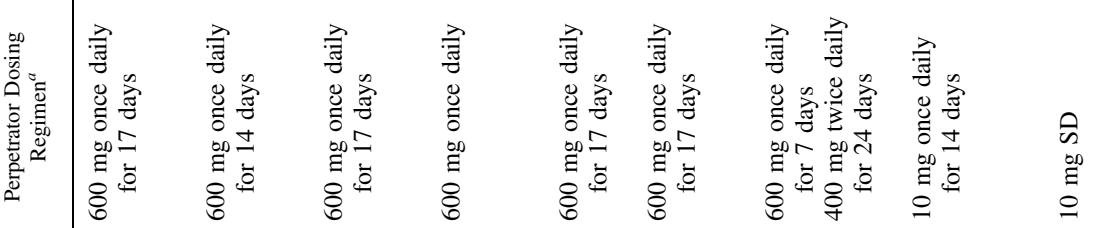

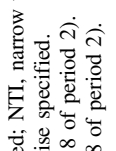

要

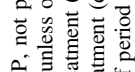

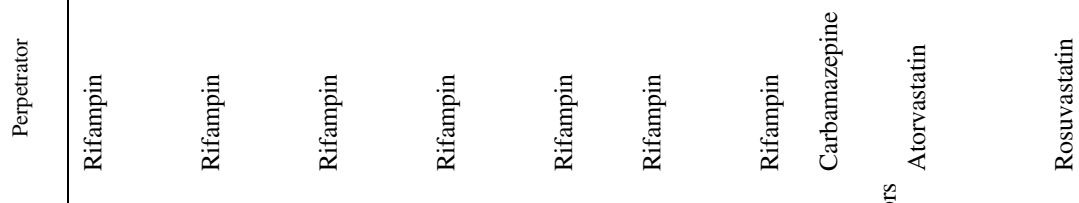

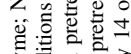

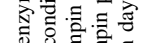

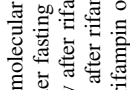

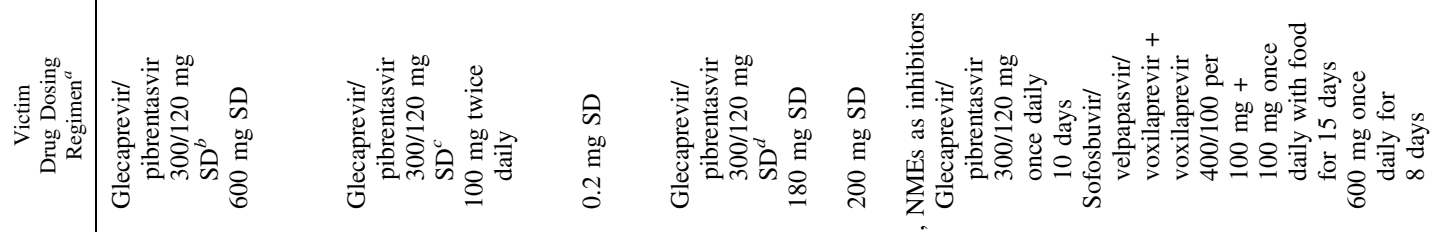

害害吉

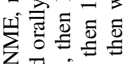


A

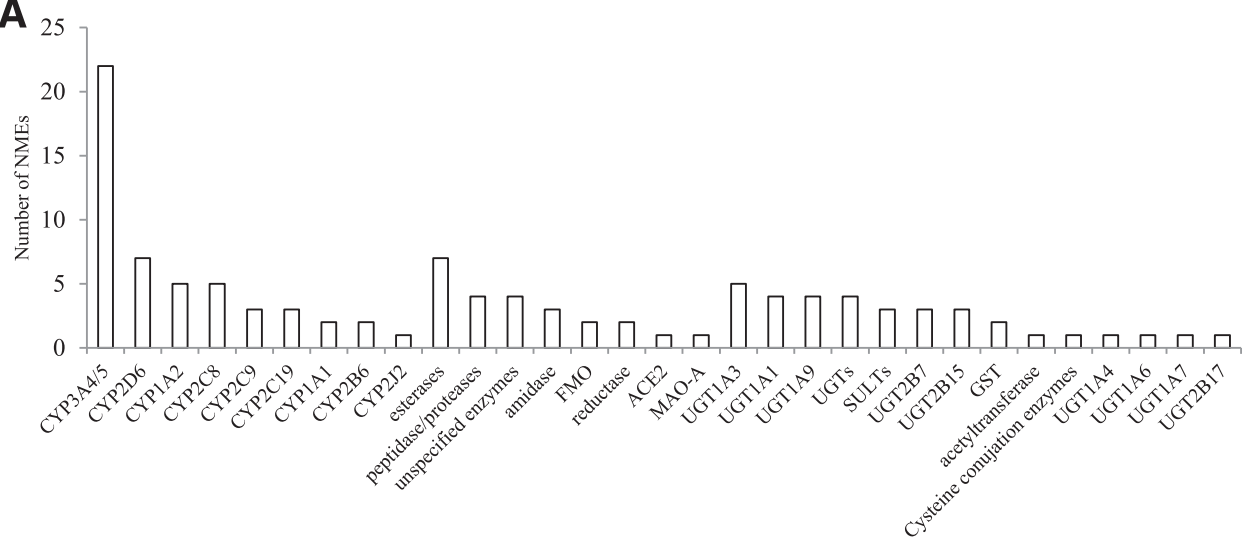

B

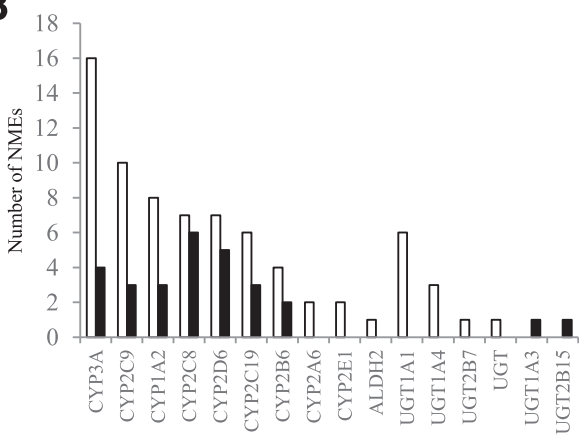

C

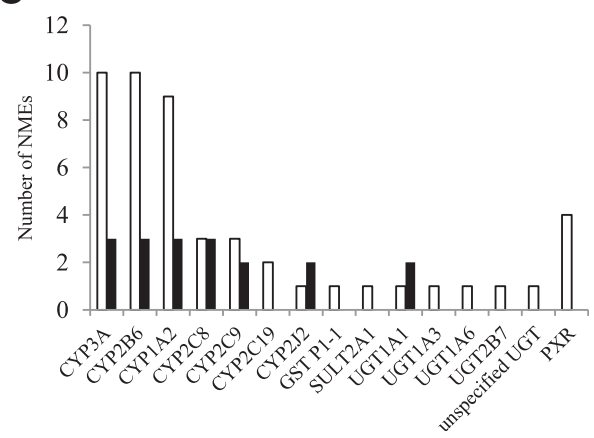

Fig. 1. The numbers of NMEs (open bars) and metabolites (closed bars) of substrates (A), inhibitors (B), or inducers (C) of drugmetabolizing enzymes. The nuclear receptor PXR is also included for induction assessment (C). ACE2, angiotensin-converting enzyme 2; ALDH2, aldehyde dehydrogenase 2; FMO, flavin-containing monooxygenase; GST, glutathione S-transferase; MAO, monoamine oxidase; SULT, sulfotransferase. adverse reactions is recommended in the label when this drug is coadministered with strong CYP3A inhibitors (FDA, 2017m).

Finally, for neratinib, a 5-fold increase in AUC was observed when it was coadministered with ketoconazole (400 mg once daily for 5 days) in healthy subjects (FDA, 2017k). The potential drug interaction risk with less potent CYP3A inhibitors was not investigated, but based on the result with ketoconazole, the label recommends that concomitant use of neratinib with both strong and moderate CYP3A inhibitors should be avoided. The sponsor was required to conduct a postmarketing evaluation (PBPK modeling and simulation or a clinical PK trial) of the effect of moderate CYP3A inhibitors on the PK of neratinib and its active metabolites.

Compared with inhibition results, more drugs were sensitive to induction with AUC changes $\geq 5$-fold ( $n=8 \mathrm{NMEs)} \mathrm{with} \mathrm{some} \mathrm{drug}$ exposures almost completely abolished by concomitant administration with the strong inducer rifampin (Table 1). Here also, drugs for cancer treatment are predominant, six of which are kinase inhibitors. All the induction interactions with AUC changes $\geq 5$-fold were also mediated by CYP3A under coadministration with rifampin or carbamazepine. As expected, abemaciclib, midostaurin, and neratinib, found to be sensitive to inhibition, were also sensitive to induction, with drug exposures significantly reduced by $90 \%-95 \%$ upon coadministration with rifampin (600 mg once daily for 8 or 14 days). Consequently, concomitant administration of these drugs with strong CYP3A inducers should be avoided (FDA, 2017k,m,p). In addition, similar changes in drug exposure $(80 \%-95 \%$ decrease) were observed or predicted for the following five drugs: acalabrutinib, brigatinib, deflazacort, naldemedine, and ribociclib; and the same labeling recommendations were included for concomitant use with strong CYP3A inducers (FDA, 2017c,f,g,i,o). In vitro studies suggest that all eight drugs are eliminated mainly by hepatic metabolism, primarily by CYP3A. Except midostaurin, they are also substrates of P-glycoprotein (P-gp), and brigatinib is also metabolized by CYP2C8 (FDA, 2017c,f,g,i,k,m,o,p). Therefore, it is possible that multiple mechanisms were involved in the induction interactions by rifampin and carbamazepine.

CYP3A played a predominant role in these significant inhibition and induction interactions. Specifically, of 12 sensitive substrates of cytochromes $\mathrm{P} 450$ (P450s, eight drugs $(67 \%)$ were sensitive substrates of CYP3A (AUC ratios $\geq 5$ in the presence of strong CYP3A inhibitors), and CYP3A was involved in all the induction interactions with AUC ratios $\leq 0.2$ (Yu et al., 2018).

For inhibition DDIs with AUC ratios of 1.25-5.0 (35 DDIs with 12 NMEs involved), most of the interactions happened with the preceding eight NMEs when coadministered with CYP3A inhibitors with different potencies. In addition, valbenazine showed a 2-fold increase in both the parent and its active metabolite $\alpha$-dihydrotetrabenzine upon coadministration with ketoconazole, suggesting that it is a moderate sensitive substrate of CYP3A (FDA, 2017h). Among the 35 DDIs, only two interactions were not mediated by CYP3A, with CYP2D6 and UDP-glucuronosyltransferase (UGT) 1A9/2B7 involved in the interactions with deutetrabenazine and ertugliflozin, respectively. Deutetrabenazine was identified as a moderate sensitive substrate of CYP2D6, with an approximate 3-fold increase in the exposure of the total active metabolites $\alpha$ - and $\beta$-deutetrabenazine when coadministered with paroxetine, a strong CYP2D6 inhibitor (FDA, 2017d). This result is consistent with pharmacogenetic studies where it was found that the total $\alpha$ - and $\beta$-deutetrabenazine exposure in subjects with impaired CYP2D6 function (poor metabolizers) was 2-fold greater than that in subjects with functional CYP2D6 (intermediate and extensive metabolizers). For ertugliflozin, in vitro studies showed that it is metabolized by UGT1A9 and UGT2B7. PBPK models predicted a 1.51-fold increase in its exposure when coadministered with mefenamic acid, a UGT inhibitor; however, a pooled analysis of ertugliflozin AUC values from 417 subjects showed that the impact of UGT1A9 allelic variants on AUC were within $90 \%-110 \%$ of the wild type and were not considered clinically relevant (FDA, 2017n). As expected, almost all the DDIs with AUC 
ratios of 2:5 triggered dose recommendations in the labels, such as avoidance, dose reduction, and monitoring for adverse reactions, whereas for DDIs with exposure changes less than 2-fold, only about half of the interactions had a labeling impact like dose reduction or monitoring for potential adverse reactions.

NMEs as Inhibitors of Enzymes. After the 2012 FDA DDI guidance recommendations for in vitro evaluation, 32 parent drugs and 24 metabolites (including the active moieties of three prodrugs) were tested for their inhibition potential on drug-metabolizing enzymes. The numbers of NMEs with positive results are presented in Fig. 1B. The largest number of drugs were inhibitors of CYP3A $(n=16)$, followed by CYP2C9, CYP1A2, CYP2C8, CYP2D6, CYP2C19, and CYP2B6.

Clinically, approximately 40 DDIs involving five drugs $(\mathrm{NMEs}=6$, with one combination of two NMEs) had AUC ratios $\geq 1.25$, including three antivirals, one cancer treatment agent, and one central nervous system agent. Ribociclib (breast cancer treatment) was the only strong inhibitor (Table 1$)$. In vitro, ribociclib showed reversible $\left(K_{\mathrm{i}, \mathrm{u}}=30.0\right.$ $\mu \mathrm{M})$ and mechanism-based inhibition $\left(K_{\mathrm{I}, \mathrm{u}}=4.44 \mu \mathrm{M}, k_{\text {inact }}=0.02 / \mathrm{min}\right)$ of CYP3A. Based on the basic model (FDA, 2012), ribociclib was predicted to inhibit CYP3A at clinically relevant concentrations $\left(C_{\max }=\right.$ $4 \mu \mathrm{M}$ at $600 \mathrm{mg}$ once daily in cancer patients). Indeed, according to PBPK models, ribociclib was predicted to increase midazolam AUC 5.17-fold at clinical doses of $600 \mathrm{mg}$ once daily for 8 days, suggesting that it is a strong inhibitor of CYP3A. In agreement with that prediction, when ribociclib was administered to healthy subjects at a lower dose of $400 \mathrm{mg}$ once daily for 8 days, a 3.89-fold increase in midazolam AUC was observed. Based on these results, caution is recommended in the label when ribociclib is coadministered with CYP3A substrates with narrow therapeutic indices (NTI). Also, the dose of a sensitive CYP3A substrate may need to be reduced (FDA, 2017i).

CYP3A was also the primary enzyme-mediating moderate and weak enzyme inhibitions (37 DDIs with five drugs involved), and only a few DDIs were mediated by CYP2C8, CYP1A2, and UGT1A1 individually or in combination with other enzymes or transporters. Based on the FDA classification, several drugs were identified as moderate or weak inhibitors of P450s through evaluations with index substrates. For example, letermovir was a moderate inhibitor of CYP3A (midazolam AUC ratio = 3.44), and glecaprevir/pibrentasvir and safinamide were weak inhibitors of CYP1A2 (caffeine AUC ratios of 1.35 and 1.30, respectively). Of note, about $20 \%$ of inhibition interactions could be attributed to inhibition of both enzymes and transporters, some mediated mainloy by enzymes and some mainly by transporters; however, the complex interplay between enzymes and transporters makes it challenging to determine the exact contribution of each mechanism. All DDIs with AUC changes of 2- to 5-fold triggered labeling recommendations, such as dose reduction, monitoring, or caution. In contrast, among all the DDIs with AUC changes less than 2-fold, only the interactions of letermovir with repaglinide or rosiglitazone (both antidiabetic agents), predicted using PBPK models, were considered clinically meaningful, and it was recommended that glucose plasma concentrations be monitored closely upon coadministration (FDA, 20171).

NMEs as Inducers of Enzymes. The induction potential of most NMEs on drug-metabolizing enzymes was systemically evaluated in vitro using human hepatocytes. A total of 31 parent drugs and 15 metabolites were assessed for their induction potential of P450s and phase 2 enzymes (glutathione S-transferases, sulfotransferases, and UGTs). Regulation of pregnane X receptor (PXR) was investigated for a few drugs. The numbers of NMEs with positive induction results are presented in Fig. 1C. Comparable numbers of drugs were inducers of CYP3A $(n=10)$, CYP2B6 $(n=10)$, and CYP1A2 $(n=9)$. Most of the induction effects, however, were observed at drug concentrations far greater than the drug's $C_{\max }$ values; based on predictions using basic or mechanistic models, these effects were not considered clinically relevant, and therefore no clinical studies were warranted. In addition, some drugs exhibited both induction and inhibition toward the same $\mathrm{P} 450$ in vitro. For example, letermovir induced CYP2B6 at concentrations up to $20 \mu \mathrm{M}$ (mRNA expression, 1.0- to 2.4-fold and $1 \%-8 \%$ of positive control; activity, 2.4- to 2.7-fold and $29-65 \%$ of positive control), whereas weak inhibition of CYP2B6 was also observed in vitro $\left(\mathrm{IC}_{50}=54 \mu \mathrm{M}\right)$. Therefore, the overall impact on CYP2B6 was expected to be minimal.

Only three drugs showed in vivo induction of enzymes, with two DDIs possibly mediated by CYP3A (telotristat ethyl and safinamide) and one by CYP2C9/2C19 (letermovir). No drug behaved as a strong clinical inducer. The largest effect was induction of CYP3A by telotristat ethyl (prodrug), with a $51 \%$ decrease observed in midazolam AUC. Although no in vitro induction of CYP3A mRNA expression was observed with telotristat ethyl concentrations of 3 and $10 \mu \mathrm{M}$, a concentration-dependent increase in PXR activation by telotristat ethyl (5.3-fold and $17.8 \%$ of the positive control rifampin at $50 \mu \mathrm{M}$ ) and telotristat (6.5-fold and $23.1 \%$ of the positive control, rifampin, at $50 \mu \mathrm{M}$ ) was observed. Induction of UGTs might also be involved as the exposure to the active metabolite 1'hydroxymidazolam similarly decreased by $49 \%$, possibly owing to increased glucuronidation of 1'-hydroxymidazolam by UGTs. In vitro induction of UGTs by telotristat ethyl, however, was not evaluated, and such studies have been requested as a postmarketing commitment (FDA, 2017s). The second largest induction was caused by letermovir, decreasing the AUC of voriconazole by $44 \%$. According to the sponsor, the induction was attributable to CYP2C9 and/or CYP2C19; however, no induction of CYP2C19 mRNA expression or activity was observed at letermovir concentrations up to $1.75 \mu \mathrm{M}$ (of note, this test concentration is far below $C_{\max }$, which is 23 and $50 \mu \mathrm{M}$ after oral and intravenous dosing of letermovir, respectively), and the induction potential of letermovir on CYP2C9 was not evaluated in vitro. These clinical study results suggest that concomitant use of telotristat ethyl or letermovir may decrease the efficacy of drugs that are substrates of CYP3A or CYP2C9/2C19 by decreasing their systemic exposure by approximately $50 \%$, respectively. Therefore, it was recommended that the plasma concentrations of victim drugs that are substrates of these enzymes be monitored and their dosages adjusted, if necessary (FDA, 20171,s).

NMEs as Substrates of Transporters. Twenty-seven NMEs and 14 metabolites were evaluated in vitro as substrates of a total of 17 transporters, and 19 parent drugs and 6 metabolites were substrates of nine transporters (Fig. 2A). The largest number of drugs were substrates of P-gp $(n=18)$, followed by breast cancer resistance protein (BCRP), organic anion transporting polypeptide (OATP) 1B1, and OATP1B3.

Approximately 20 clinical DDI studies found AUC ratios $\geq 1.25$ (with five drugs involved) that were mediated mainly by transporters, namely, OATP1B1, OATP1B3, P-gp, and BCRP. OATP1B1/1B3 played a dominant role, mediating more than half of these DDIs, some with involvement of P-gp and BCRP. As discussed in the enzyme section, almost all the moderate sensitive and sensitive substrates of CYP3A are also substrates of P-gp and, to avoid redundancy, these interactions are not included in this section. Among the five drugs (NMEs $=6$, including one combination drug with two NMEs) involved, three drugs (NMEs $=4$ ) are antivirals. Approximately $80 \%$ of the DDIs were from two drugs, glecaprevir/pilbentasvir and voxilaprevir, both indicated for the treatment of hepatitis $\mathrm{C}$ virus (HCV) infection. About half of the interactions were comedication studies pwing to the complex polypharmacy in this patient population.

Five drug interaction studies (involving two NMEs) had AUC changes greater than 5-fold, all mediated by OATP1B1/1B3, with partial contributions from P-gp and BCRP for some of those interactions (Table 1). Based on these inhibition study results, two NMEs, namely, glecaprevir and voxilaprevir, were identified as sensitive substrates of OATP1B1/1B3. Coadministration of voxilaprevir in healthy subjects with cyclosporine (600 mg oral single dose), an inhibitor of 

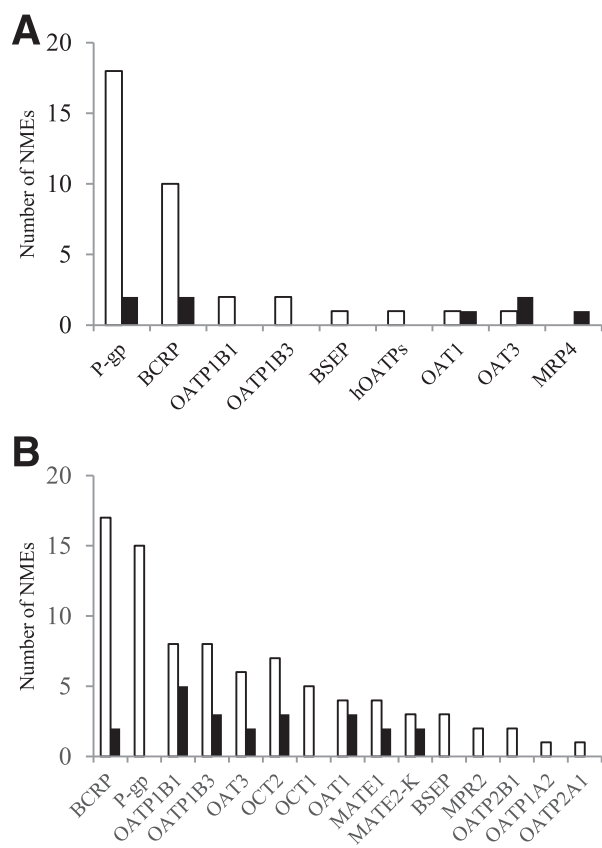

Fig. 2. The numbers of NMEs (open bars) and metabolites (closed bars) of substrates (A) or inhibitors (B) of drug transporters. BSEP, bile salt export pump; MRP, multidrug resistance-associated protein.

OATP1B1/1B3 (also of other transporters), significantly increased voxilaprevir AUC by approximately 10-fold. Similarly, a 600-mg single-dose oral administration of rifampin in healthy subjects resulted in an 8-fold increase in voxilaprevir AUC, suggesting strong inhibition of OATP1B1/1B3. Some in vitro evidence suggests that uptake transporters are involved in the transport of voxilaprevir in hepatocytes, although specific transporters were not identified. Voxilaprevir is also a substrate of P-gp and BCRP, with efflux ratios of 6.5 and 3.9, respectively, in transporter-transfected Madin-Darby Canine Kidney II cells (FDA, 2017q). Therefore, the greater change in voxilaprevir AUC observed with cyclosporine may involve inhibition of P-gp and BCRP as cyclosporine is also an inhibitor of these two transporters. Regarding glecaprevir, its exposure was increased by 8.55 - and 5.08-fold in healthy subjects under coadministration with a single oral dose of rifampin (600 mg) or cyclosporine (400 mg), respectively. Like voxilaprevir, glecaprevir is a substrate of OATP1B1 $\left(K_{\mathrm{m}}=0.098 \mu \mathrm{M}\right)$, OATP1B3 $\left(\mathrm{K}_{\mathrm{m}}=0.19 \mu \mathrm{M}\right), \mathrm{P}-\mathrm{gp}($ efflux ratio $=7.8)$, and BCRP $($ efflux ratio $=9.3)$ (FDA, 2017j). As expected, based on the clinical findings, concomitant administration of glecaprevir or voxilaprevir with OATP inhibitors is contraindicated or not recommended (FDA, 2017j,q).

For DDIs with AUC changes 1.25- to 5-fold, OATP1B1/1B3 was also primarily involved, mediating two-thirds of the interactions. For example, the interactions between the combination drug glecaprevir or pibrentasvir and some commonly prescribed comedications, such as atazanavir or ritonavir, cobicistat, darunavir or ritonavir, and lopinavir or ritonavir were mediated mainly by OATP1B1/1B3, with possible involvement of P-gp and/or BCRP. Although inhibition of P-gp seems to contribute to all the drug interactions with AUC ratios of 1.25-5.0, it was the main driving mechanism in only three DDIs involving two NMEs: 1) coadministration of betrixaban with verapamil and ketoconazole showed 3.06- and 2.12-fold increases in the exposure of betrixaban; 2) naldemedine AUC increased 1.79-fold when it was coadministered with cyclosporine. Based on these results, it is recommended that the dose of betrixaban be reduced or monitored for potential naldemedine-related adverse reactions when they are coadministered with P-gp inhibitors (FDA, 2017e, o).
On the other hand, several drugs seemed more sensitive to induction than to inhibition. For example, when the combination drug glecaprevir/ pibrentasvir was coadministered with multiple oral doses of rifampin (600 mg once daily for 17 days), the AUC and $C_{\max }$ values of glecaprevir and pibrentasvir were significantly reduced, by approximately $90 \%$, mainly because of induction of P-gp (induction of CYP3A might also contribute to the reduction in glecaprevir exposure since, in vitro, glecaprevir exhibited some metabolism, primarily by CYP3A). The less potent inducers carbamazepine and efavirenz decreased these drugs' exposure by $50 \%-70 \%$ and $50 \%$, respectively. Consequently, considering the potential risk of therapeutic failure, coadministration of glecaprevir/pibrentasvir is contraindicated with rifampin but not recommended with carbamazepine or efavirenz (FDA, 2017j).

NMEs as Inhibitors of Transporters. In vitro, the inhibition potential of NMEs and their metabolites was assessed toward 19 transporters. A total of 31 NMEs and 26 metabolites (including the active moieties of two prodrugs) were evaluated; 25 NMEs and 9 metabolites showed inhibition. The largest number of drugs was inhibitors of BCRP $(n=17)$, followed by P-gp $(n=15)$, OATP1B1 $(n=8)$, and OATP1B3 $(n=8)$ (Fig. 2B).

Clinically, more than 20 DDIs occurred involving six drugs $(\mathrm{NMEs}=$ 7 , including one combination drug with two NMEs) with AUC ratios $\geq$ 1.25 , with about a third of the interactions mediated by both transporters and enzymes. Among these inhibitors are three antivirals, two cancer treatment agents, and one central nervous system agent. Even though OATP1B1 and OATP1B3 were not the main transporters inhibited in vitro, they played a dominant role in vivo, mediating half of the drug interactions including all the strong interactions (two DDIs; Table 1) and most of the moderate ones (six of nine DDIs).

Two drugs, glecaprevir/pibrentasvir and sofosbuvir/velpatasvir/ voxilaprevir, both HCV combination drugs, exhibited strong inhibition of OATP1B1/1B3 and/or BCRP, with greater than 5-fold increase in the exposure of victim drugs atorvastatin or rosuvastatin (Table 1). Coadministration with glecaprevi/pibrentasvir (300 mg/120 mg once daily for 10 days) in healthy subjects significantly increased the AUC and $C_{\max }$ of atorvastatin, a clinical substrate of OATP1B1/1B3, 8.28- and 22-fold, respectively. In vitro, glecaprevir inhibited OATP1B1 $\left(\mathrm{IC}_{50}=0.017 \mu \mathrm{M}\right)$ and OATP1B3 $\left(\mathrm{IC}_{50}=0.064 \mu \mathrm{M}\right)$, and pibrentasvir inhibited OATP1B1 $\left(\mathrm{IC}_{50}=1.3 \mu \mathrm{M}\right.$ with $4 \%$ bovine serum albumin), but not OATP1B3 $\left(\mathrm{IC}_{50}>30 \mu \mathrm{M}\right)$. Of note, glecaprevir also weakly inhibited CYP3A in vitro $\left(\mathrm{IC}_{50}=28.4 \mu \mathrm{M}\right)$, and atorvastatin is a moderate sensitive substrate of CYP3; therefore, inhibition of CYP3A might also contribute to the overall effect. Considering the large increase in atorvastatin exposure, coadministration of glecaprevir/pibrentasvir with atorvastatin is not recommended (FDA, 2017j). The drug interaction risks with other statins (lovastatin, pravastatin, rosuvastatin, simvastatin) were evaluated as well. Exposures to these statins were increased to a much smaller extent (1.70- to 2.32-fold) in healthy subjects upon coadministration with gelcaprevir/pibrentasvir; however, there was a greater increase (4.10- to 4.50 -fold) in the active metabolites lovastatin hydroxyl acid and simvastatin acid. Therefore, coadministration of lovastatin or simvastatin with glecaprevir/pibrentasvir is also not recommended; dose reduction is recommended for pravastatin and rosuvastatin (FDA, 2017j).

The other strong inhibition was caused by the combination drug sofosbuvir/velpatasvir/voxilaprevir (400 mg-100 mg-100 mg + $100 \mathrm{mg}$ voxilaprevir once daily for 15 days; voxilaprevir as an NME), with 7.35and 17.96-fold increases in the AUC and $C_{\max }$ of coadministered rosuvastatin, respectively, a clinical substrate of BCRP, OATP1B1, and OATP1B3 (FDA, 2017q). In vitro studies showed that voxilaprevir is an inhibitor of OATP1B1 $\left(\mathrm{IC}_{50}=0.18 \mu \mathrm{M}\right)$ and OATP1B3 $\left(\mathrm{IC}_{50}=\right.$ $0.70 \mu \mathrm{M}$ ), and velpatasvir inhibits BCRP, OATP1B1, and OATP1B3 with $\mathrm{IC}_{50}$ values of $0.30,1.5$, and $0.26 \mu \mathrm{M}$, respectively. Sofosbuvir only weakly inhibited BCRP (35\% inhibition at $100 \mu \mathrm{M})$ and OATP1B3 
Yu et al.

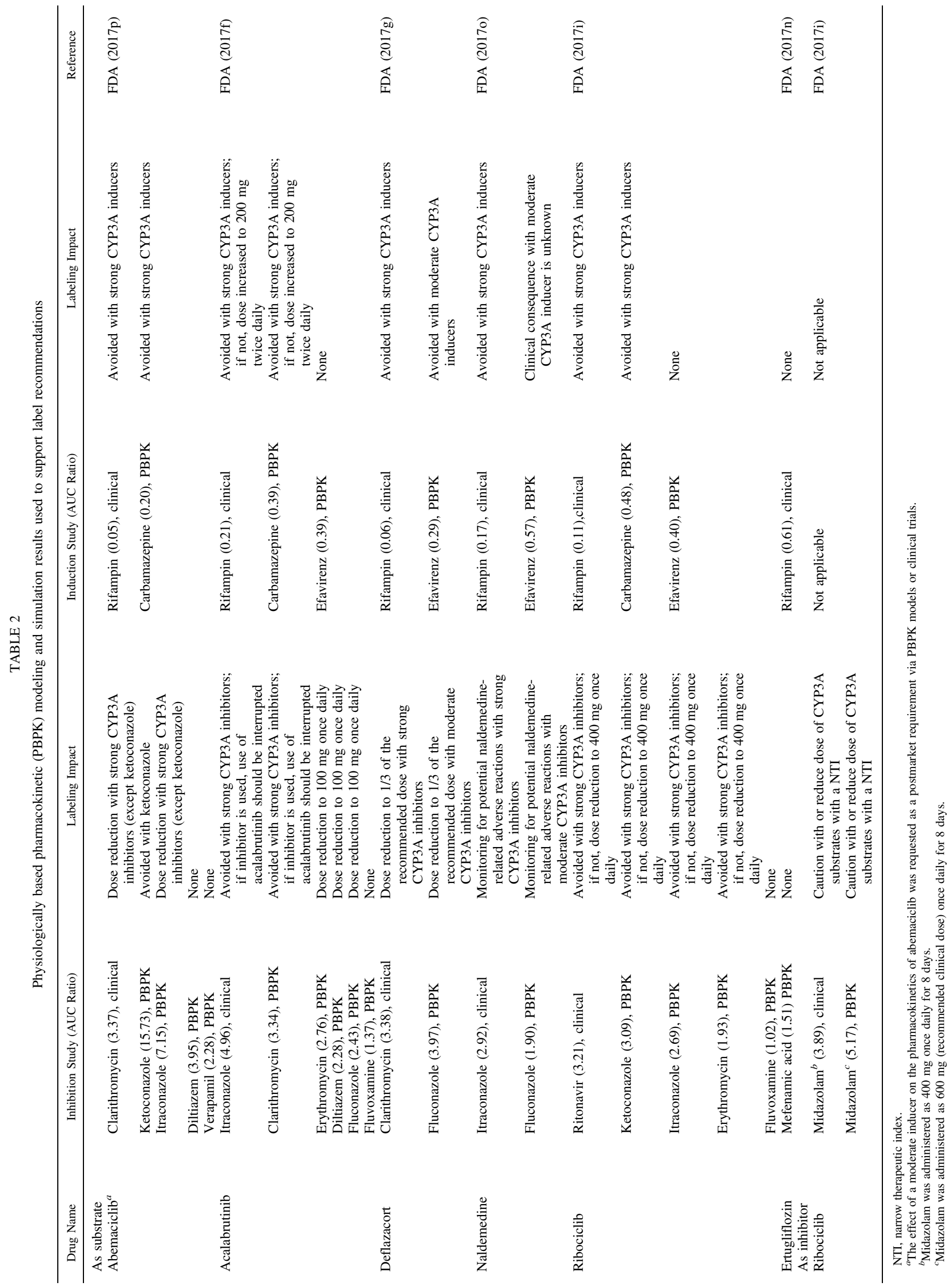

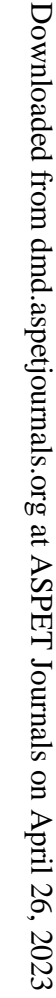


$\left(\mathrm{IC}_{50}=203.5 \mu \mathrm{M}\right)$. Based on in vitro-to-in vivo prediction calculations (FDA, 2012), both velpatasvir and voxilaprevir were likely to inhibit these transporters at clinically relevant concentrations. In vivo, coadministration of velpatasvir (100 mg daily for 11 days) increased pravastatin AUC 1.35-fold, likely owing to inhibition of hepatic OATPs (Mogalian et al., 2016; FDA, 2017q). A larger effect was observed for rosuvastatin, with approximately a 2.8-fold increase in its AUC after coadministration of velpatasvir (100 mg daily for 11 days), owing to the inhibition of both OATPs and BCRP (Mogalian et al., 2016; FDA, 2017q). Therefore, the significant increase in rosuvastatin under coadministration with sofosbuvir/velpatasvir/voxilaprevir may be attributable to the combined inhibition of BCRP, OATP1B1, and OATP1B3 by velpatasvir and voxilaprevir.

P-gp was involved in approximately one-third of the clinical interactions, with most victim exposure changes less than 2-fold. The largest effect mediated by P-gp was observed with the combination drug sofosbuvir/velpatasvir/voxilaprevir, increasing dabigatran (clinical substrate of P-gp) AUC 2.59-fold. Clinical monitoring of dabigatran is recommended when coadministered with this combination drug (FDA 2017q). Although the exposure increases of the P-gp substrate digoxin were lower (1.30-1.48) when coadministered with glecaprevir/ pibrentasvir, neratinib, or valbenazine, these effects were considered clinically relevant, and monitoring or dose reduction was recommended in the drugs' labels when used concomitantly with P-gp substrates with a NTI such as digoxin (FDA, 2017h,j,k). Regarding other transporters, abemaciclib was a weak inhibitor of multi-antimicrobial extrusion protein 1 and $2-\mathrm{K}$ and organic cation transporter 2 , as it slightly increased metformin (clinical substrate of these transporters) AUC 1.37fold; however, this effect was not considered clinically relevant. Like enzyme-mediated DDIs, almost all the interactions with AUC ratios of 2 to 5 triggered dose recommendations, whereas for DDIs with AUC ratios less than 2, most were not considered clinically meaningful.

In Vitro-to-In Vivo Predictions. According to recommendations of the 2012 FDA DDI guidance, in vitro-to-in vivo predictions were performed using both basic and mechanistic models. PBPK models were used to predict the risk of drug interactions for six drugs, and the predicted results were used to support specific label recommendations (Table 2). Among them, five drugs (abemaciclib, acalabrutinib, deflazacort, naldemedine, and ribociclib) are substrates of CYP3A. Typically, the drug interaction risk with one strong inhibitor or inducer was evaluated using a clinical study, whereas the risks with other strong inhibitors or inducers or less potent inhibitors or inducers were predicted using PBPK models. PBPK models were also used to predict the inhibition potential of different dosing regimens for ribociclib. The effect of ribociclib at $400 \mathrm{mg}$ once daily for 8 days on CYP3A was investigated in a clinical trial in healthy subjects using midazolam as the probe substrate, and the recommended dose of $600 \mathrm{mg}$ once daily for 8 days was assessed using PBPK models. As expected, a larger increase in midazolam was predicted with the higher dose of ribociclib, and label recommendations were based on those predictions (FDA, 2017i).

Basic model predictions were performed more widely for all drugs. In general, good predictions were observed for drugs that showed high inhibition or induction potency in vitro, such as ribociclib $\left(C_{\max }=4 \mu \mathrm{M}\right)$, which showed reversible $\left(K_{\mathrm{i}, \mathrm{u}}=30.0 \mu \mathrm{M}\right)$ and mechanism-based inhibition $\left(K_{\mathrm{I}, \mathrm{u}}=4.44 \mu \mathrm{M}, k_{\text {inact }}=0.02 / \mathrm{min}\right)$ of CYP3A; glecaprevir $\left(C_{\max }=0.712 \mu \mathrm{M}\right)$, which inhibited P-gp $\left(\mathrm{IC}_{50}=0.33 \mu \mathrm{M}\right)$; BCRP $\left(\mathrm{IC}_{50}=2.3 \mu \mathrm{M}\right)$, OATP1B1 $\left(\mathrm{IC}_{50}=0.017 \mu \mathrm{M}\right)$, and OATP1B3 $\left(\mathrm{IC}_{50}=\right.$ $0.064 \mu \mathrm{M}$ ); and letermovir $\left(C_{\max }=22.7 \mu \mathrm{M}\right.$ (oral), $49.6 \mu \mathrm{M}$ (i.v.)), which inhibited OATP1B1 $\left(\mathrm{IC}_{50}=2.9 \mu \mathrm{M}\right)$ and OATP1B3 $\left(\mathrm{IC}_{50}=1.1 \mu \mathrm{M}\right)$ (FDA, 2017i,j,1). Indeed, in vivo, all these drugs exhibited moderate to strong inhibition of the relevant enzyme or transporter; however, in several cases, in vitro findings did not accurately predict the clinical results using basic models. For example, betrixaban, letermovir, and telotristat ethyl all showed inhibition of P-gp in vitro. Based on the $[\mathrm{I}] / \mathrm{IC}_{50}$ ratios, betrixaban and telotristat ethyl were expected to inhibit $\mathrm{P}$-gp at the gut level, whereas letermovir had the potential to cause both systemic and intestinal inhibition of P-gp. When evaluated in vivo, however, no significant increase in the exposure of the coadministered P-gp substrates digoxin or fexofenadine (AUC ratios 0.90-1.14) were found, indicating no clinical inhibition of P-gp (FDA, 2017e,1,s). On the other hand, several drugs predicted to have a remote risk of drug interaction showed positive clinical inhibition. For instance, for the combination drug glecaprevir/pibrentasvir, glecaprevir only weakly inhibited CYP3A4 $\left(\mathrm{IC}_{50}=28.3 \mu \mathrm{M}\right)$ but not CYP1A2, and pibrentasvir did not inhibit any P450s. Based on the $R_{1}$ value of 1.05 (assuming competitive inhibition, $K_{\mathrm{i}}=14.15 \mu \mathrm{M} ; C_{\max }=0.712 \mu \mathrm{M}$ ) for CYP3A4, glecaprevir was not likely to inhibit this enzyme at clinically relevant concentrations. In a cocktail study, however, the combination exhibited weak inhibition of both CYP1A2 and CYP3A, with a 35\% and 27\% increase observed in the AUC of caffeine and midazolam, respectively (FDA, 2017j). Similarly, safinamide showed reversible $\left(\mathrm{IC}_{50}=47.7 \mu \mathrm{M}\right.$; $K_{\mathrm{i}}=54 \mu \mathrm{M}$, competitive $)$ and mechanism-based inhibition $\left(K_{\mathrm{I}}=33.5 \mu \mathrm{M}\right.$, $K_{\text {inact }}=0.075 / \mathrm{min}$ ) of CYP1A2, and its metabolite NW-1153 also showed weak inhibition of CYP1A2 (36\% inhibition under preincubation and $8.9 \%$ inhibition under coincubation). With both $R_{1}$ and $R_{2}$ values $<1.1$, no clinical inhibition of CYP1A2 was expected. However, safinamide co-administration caused a $30 \%$ increase in caffeine AUC, suggesting weak inhibition of CYP1A2 in vivo (FDA, 2017r). Although the slight increases in exposure to these probes were not considered clinically meaningful by the sponsors, based on the current FDA classification, both drugs are still considered weak inhibitors. If only the basic models were used, and no clinical studies had been performed, these weak inhibitions would not have been identified. One possible reason causing the prediction discrepancy of these basic models may be the failure to consider the drug's protein or plasma binding capacity. Of note, for the transporters P-gp, BCRP, and OATP1B1/1B3, among the 13 drugs with prediction values higher than the cutoffs, nine drugs were highly bound to plasma $(>96 \%)$. In the most recent FDA guidance $(2017 \mathrm{a}, \mathrm{b})$, the prediction methods and cutoffs have been revised, and it is now recommended that unbound inhibition parameters and free plasma concentrations be used in the calculations. Also, a preincubation condition is recommended for transporter inhibition studies; however, because the unbound inhibition parameters were not available for most of the drugs approved in 2017, it was not possible to evaluate whether prediction accuracy would have been enhanced with the new regulatory framework.

\section{Discussion and Conclusions}

The PK-based DDI data from NDA reviews for drugs approved by the FDA in 2017 were thoroughly reviewed, and the clinical significance of the results was assessed. As expected, CYP3A mediated most interactions, whether NMEs serving as substrates or as perpetrators. A total of 11 drugs were found to be clinical substrates of CYP3A, with AUC increases $\geq 25 \%$ when coadministered with a strong CYP3A inhibitor (clarithromycin, itraconazole, ketoconazole, ritonavir, or voriconazole). Among these drugs, nine (82\%) were also substrates of P-gp in vitro, consistent with previous findings and confirming the significant overlap between substrates of CYP3A and P-gp (Christians et al., 2005; Zhou 2008; Yu et al., 2018). Inhibition of OATP1B1/1B3 also played a substantial role. All large interactions with AUC changes of the victim drug equal to or greater than 5-fold were mediated by either CYP3A or OATP1B/1B3, with contributions from other transporters such as P-gp and/or BCRP. Five drugs were considered sensitive substrates, including abemeciclib, midostaurin, and neratinib for CYP3A and glecaprevir and 
voxilaprevir for OATP1B1/1B3. When NMEs were evaluated as perpetrators, three drugs were considered strong inhibitors, including ribociclib for CYP3A, glecaprevir/pibrentasvir for OATP1B1/1B3, and sofosbuvir/velpatasvir/voxilaprevir for OATP1B1/1B3 and BCRP. No strong inducer was identified.

As noted in previous evaluations (Yu et al., 2018), transporters are playing an increasing role in clinical drug interactions. This is mostly explained by an improved ability to detect transporter-mediated DDIs because of a better understanding of their role in drug disposition, as well as deliberate efforts to shift molecular structures away from P450mediated interactions during the early phase of drug discovery (Cheng et al., 2007; Hughes et al., 2011). For the 2017 NMEs, they mediated about half of all drug interactions as main or partial contributors to the overall effect, with often complex interplays observed between several transporters or between transporters and enzymes, particularly for DDIs involving antiviral combination drugs. Two combination drugs, glecaprevir/pibrentasvir and sofosbuvri/velpatasvir/voxilaprevir, were identified as both sensitive substrates and strong inhibitors of transporters. Despite our improved knowledge of the underlying mechanisms of DDIs, because of the complexity of the enzyme-transporter interplay, understanding the true contribution of a transporter in a specific interaction remains challenging because of the lack of specific transporter substrates and inhibitors. Also, it is often difficult to generalize transporter-based DDIs among substrates and inhibitors.

In terms of labeling impact, all large DDIs with exposure changes equal to or greater than 5-fold triggered labeling recommendations such as contraindication, not recommended, or avoidance of concomitant administration. Almost all drug interactions with AUC changes of 2- to 5-fold also had some language in the labels recommending dose adjustment of the victim drug or monitoring for adverse reactions or plasma concentrations. For DDIs with exposure changes of less than 2-fold, however, there were differences in labeling impact, depending on the NME's role in the drug interaction. When NMEs were victims, approximately half of the drug interactions had some language in the label like dose adjustment or monitoring, whereas only about $20 \%$ of DDIs triggered labeling recommendations when NMEs were the perpetrators. In both cases, labeling recommendations were mainly related to NTI drugs. Interestingly, almost all induction DDIs with AUC changes less than 5-fold (NMEs as substrates) had labeling language to avoid coadministration with inducers given the impact of possible loss of efficacy for these classes of drugs.

Finally, it is noteworthy that, as observed in previous years, the largest clinical interactions involving NMEs identified as sensitive substrates and strong inhibitors were observed with oncology or HCV drugs, including four (of the nine approved) oncology drugs and two (out of three approved) antiviral drugs. This finding suggests a significant risk of clinically significant DDIs in these patient populations for whom therapeutic management is already complex owing to polytherapy.

\section{Acknowledgments}

We thank Dr. Sophie Argon, Dr. Katie H. Owens, and Dr. Catherine K. Yeung for their contributions to the NDA data curation.

\section{Authorship Contributions}

Participated in research design: Yu, Levy, Ragueneau-Majlessi.

Performed data analysis: Yu, Petrie, Levy, Ragueneau-Majlessi.

Wrote or contributed to the writing of the manuscript: Yu, Petrie, Levy, Ragueneau-Majlessi.

\section{References}

Abbas R, Hug BA, Leister C, Burns J, and Sonnichsen D (2011) Pharmacokinetics of oral neratinib during co-administration of ketoconazole in healthy subjects. Br J Clin Pharmacol 71:522-527. Boulenc X, Nicolas O, Hermabessière S, Zobouyan I, Martin V, Donazzolo Y, and Ollier C (2016) CYP3A4-based drug-drug interaction: CYP3A4 substrates' pharmacokinetic properties and ketoconazole dose regimen effect. Eur J Drug Metab Pharmacokinet 41:45-54.
Boxenbaum H (1999) Cytochrome P450 3A4 in vivo ketoconazole competitive inhibition: determination of $\mathrm{Ki}$ and dangers associated with high clearance drugs in general. J Pharm Pharm Sci 2:47-52.

Cheng KC, Korfmacher WA, White RE, and Njoroge FG (2007) Lead optimization in discovery drug metabolism and pharmacokinetics/case study: the hepatitis $\mathrm{C}$ virus (HCV) protease inhibitor SCH 503034. Perspect Medicin Chem 1:1-9.

Christians U, Schmitz V, and Haschke M (2005) Functional interactions between P-glycoprotein and CYP3A in drug metabolism. Expert Opin Drug Metab Toxicol 1:641-654.

Dutreix C, Munarini F, Lorenzo S, Roesel J, and Wang Y (2013) Investigation into CYP3A4mediated drug-drug interactions on midostaurin in healthy volunteers. Cancer Chemother Pharmacol 72:1223-1234.

Food and Drug Administration (FDA) (2012) Guidance for industry: drug interaction studiesstudy sesign, data analysis, and implications for dosing and labeling recommendations (draft guidance), Center for Drug Evaluation and Research., FDA, Silver Spring, MD.

Food and Drug Administration (FDA) (2017a) Guidance for industry: clinical drug interaction studies - study design, data analysis, and clinical implications for dosing and labeling recommendations (draft guidance), Center for Drug Evaluation and Research., FDA, Silver Spring, MD.

Food and Drug Administration (FDA) (2017b) Guidance for industry: in vitro metabolism- and transporter-mediated drug-drug interaction studies (draft guidance). Center for Drug Evaluation and Research., FDA, Silver Spring, MD.

Food and Drug Administration (FDA) (2017c) Drug Approval Package: ALUNBRIG (Brigatinib). FDA Application NDA 208772, FDA, Silver Spring, MD.

Food and Drug Administration (FDA) (2017d) Drug Approval Package: AUSTEDO (Deutetrabenazine). FDA Application NDA 208082, FDA, Silver Spring, MD.

Food and Drug Administration (FDA) (2017e) Drug Approval Package: BEVYXXA (Betrixaban). FDA Application NDA 208383, FDA, Silver Spring, MD.

Food and Drug Administration (FDA) (2017f) Drug Approval Package: CALQUENCE (Acalabrutinib). FDA Application NDA 210259, FDA, Silver Spring, MD.

Food and Drug Administration (FDA) (2017g) Drug Approval Package: EMFLAZA (Deflazacort). FDA Application NDA 208684, FDA, Silver Spring, MD.

Food and Drug Administration (FDA) (2017h) Drug Approval Package: INGREZZA (Valbenazine) FDA Application NDA 209241, FDA, Silver Spring, MD.

Food and Drug Administration (FDA) (2017i) Drug Approval Package: KISQALI (Ribociclib Succinate). FDA Application NDA 209092, FDA, Silver Spring, MD.

Food and Drug Administration (FDA) (2017j) Drug Approval Package: MAVYRET (Glecaprevir and Pibrentasvir). FDA Application NDA 209394, FDA, Silver Spring, MD.

Food and Drug Administration (FDA) (2017k) Drug Approval Package: NERLYNX (Neratinib Maleate). FDA Application NDA 208051, FDA, Silver Spring, MD.

Food and Drug Administration FDA (2017l) Drug Approval Package: PREVYMIS (Letermovir). FDA Application NDA 209939, FDA, Silver Spring, MD.

Food and Drug Administration FDA (2017m) Drug Approval Package: RYDAPT (Midostaurin). FDA Application NDA 207997, FDA, Silver Spring, MD.

Food and Drug Administration FDA (2017n) Drug Approval Package: STEGLATRO (Ertugliflozin). FDA Application NDA 209803, FDA, Silver Spring, MD.

Food and Drug Administration FDA (2017o) Drug Approval Package: SYMPROIC (Naldemedine Tosylate). FDA Application NDA 208854, FDA, Silver Spring, MD.

Food and Drug Administration FDA (2017p) Drug Approval Package: VERZENIO (Abemaciclib). FDA Application NDA 208716, FDA, Silver Spring, MD.

Food and Drug Administration FDA (2017q) Drug Approval Package: VOSEVI (Sofosbuvir Velpatasvir, and Voxilaprevir). FDA Application NDA 209195, FDA, Silver Spring, MD.

Food and Drug Administration (FDA (2017r) Drug Approval Package: XADAGO (Safinamide). FDA Application NDA 207145, FDA, Silver Spring, MD

Food and Drug Administration (FDA) (2017s) Drug Approval Package: XERMELO (Telotristat Ethyl). FDA Application NDA 208794, FDA, Silver Spring, MD.

Food and Drug Administration (FDA) (2018) Guidance for industry: physiologically based pharmacokinetic analyses-format and content, Center for Drug Evaluation and Research, FDA, Silver Spring, MD

Gurley B, Hubbard MA, Williams DK, Thaden J, Tong Y, Gentry WB, Breen P, Carrier DJ, and Cheboyina S (2006) Assessing the clinical significance of botanical supplementation on human cytochrome P450 3A activity: comparison of a milk thistle and black cohosh product to rifampin and clarithromycin. J Clin Pharmacol 46:201-213.

Hughes JP, Rees S, Kalindjian SB, and Philpott KL (2011) Principles of early drug discovery. Br J Pharmacol 162:1239-1249.

Ito K, Brown HS, and Houston JB (2004) Database analyses for the prediction of in vivo drug-drug interactions from in vitro data. Br J Clin Pharmacol 57:473-486.

Mogalian E, German P, Kearney BP, Yang CY, Brainard D, McNally J, Moorehead L, and Mathias A (2016) Use of multiple probes to assess transporter- and cytochrome P450-mediated drug-drug interaction potential of the pangenotypic HCV NS5A inhibitor velpatasvir. Clin Pharmacokinet 55:605-613.

Olkkola KT, Backman JT, and Neuvonen PJ (1994) Midazolam should be avoided in patients receiving the systemic antimycotics ketoconazole or itraconazole. Clin Pharmacol Ther 55:481-485.

Yu J, Ritchie TK, Mulgaonkar A, and Ragueneau-Majlessi I (2014) Drug disposition and drug-drug interaction data in 2013 FDA new drug applications: a systematic review. Drug Metab Dispos 42:1991-2001

Yu J, Ritchie TK, Zhou Z, and Ragueneau-Majlessi I (2016) Key findings from preclinical and clinical drug interaction studies presented in new drug and biological license applications approved by the Food and Drug Administration in 2014. Drug Metab Dispos 44:83-101.

Yu J, Zhou Z, Owens KH, Ritchie TK, and Ragueneau-Majlessi I (2017) What can be learned from recent new drug applications? A systematic review of drug interaction data for drugs approved by the US FDA in 2015. Drug Metab Dispos 45:86-108.

Yu J, Zhou Z, Tay-Sontheimer J, Levy RH, and Ragueneau-Majlessi I (2018) Risk of clinically relevant pharmacokinetic-based drug-drug interactions with drugs approved by the U.S. Food and Drug Administration between 2013 and 2016. Drug Metab Dispos 46:835-845.

Zhou SF (2008) Drugs behave as substrates, inhibitors and inducers of human cytochrome P450 3A4. Curr Drug Metab 9:310-322.

Address correspondence to: Jingjing Yu, Drug Interaction Database (DIDB) Program, Department of Pharmaceutics, School of Pharmacy, University of Washington, Box 357610, Seattle, WA 98195. E-mail: jingyu@uw.edu 